\title{
Alternativas a la enseñanza tradicional de la lectura
}

\author{
J. Lloyd Eldredge \\ Dennie Butterfield
}

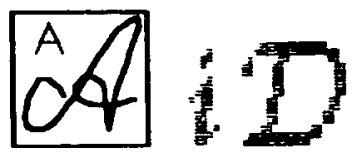

a investigación sobre las estrategias didácticas más apropiadas para la enseñanza de la lectura no siempre se ocupa de las consecuencias prácticas sobre la organización de la clase que implica cada una.

En este artículo, se comparan estrategias clásicas de enseñanza de la lectura con otras nuevas, con una clara y precisa definición de los métodos a seguir en aquellas que resultan más eficaces.

El recién publicado informe de la Comisión para la lectura de los Estados Unidos (Anderson et al., 1985) ofrece razones apremiantes para realizar cambios radicales en la forma en que se enseña la lectura en la escuela. El informe pone en cuestión muchas prácticas y materiales tradicionales, como agrupamiento de los alumnos según su capacidad, los textos básicos de lectura y la enseñanza de silabeo implícita. Este artículo propone algunas alternativas recientemente implementadas y sometidas a verificación.

\section{ALTERNATIVAS AL AGRUPAMIENTO POR CAPACIDAD, A LOS PROGRAMAS DE LECTURA BASICA Y AL SILABEO}

Hace unos pocos años, decidimos probar la eficacia de formas alternativas para enseñar a leer a los niños pequeños. Habíamos pasado muchos años en la administración de colegios públicos como director o inspector antes de convertirnos en formadores de profesores. También nos habíamos sentido frustrados ante los problemas administrativos que se derivaban de niños situados en grupos de lectura de "bajo nivel» para la enseñanza de la lectura. Observamos que los niños situados en los grupos de lectura de bajo

*Alternatives to traditional reading instruction. The Reading Teacher, 1986, 40 (I), pp. 32-37. Reproducido con autorización de J. Lloyd Eldredge y de la International Reading Association (C). Traducción al castellano CLEE (Traducción de Carmen Trillo. La I.R.A. no se responsabiliza de la adecuación de la traducción). 
nivel durante el primer curso se solían mantener en los mismos grupos inferiores en sexto curso y que, incluso entonces, los problemas no parecían tener fin. Esta común experiencia nos sirvió de catalizador para un proyecto de investigación diseñado para constatar si los niños podrían aprender o no a leer eficazmente sin utilizar el agrupamiento por capacidad.

La preocupación por la gran cantidad de tiempo invertido en la ensenanza del silabeo en los primeros cursos y en el método analítico de lectura básica, fue la motivación decisiva que nos impulsó a probar la eficacia de las alternativas existentes a los programas básicos y a la enseñanza tradicional del silabeo.

En un principio nos hicimos tres sencillas preguntas: 1. ¿Pueden los niños aprender a leer en grupos heterogéneos donde todos los niños de la clase utilizan el mismo texto básico de lectura tan bien como aprenden a leer en grupos igualados por capacidad? 2. ¿Pueden los niños aprender a leer utilizando libros de literatura infantil tan bien como aprenden a leer utilizando el libro básico? 3. ¿Será tan efectiva una aproximación alternativa a la decodificación que requiera menos tiempo de clase y de dedicación por parte del profesor como las estrategias de decodificación que se utilizan en programas básicos de lectura?

Se implementaron cinco enfoques alternativos en cuatro distritos escolares de Utah para contestar a las preguntas de la investigación. Los sujetos eran 1.149 niños de 50 clases de 24 colegios; se utilizaron 26 clases para probar la eficacia de los cinco enfoques alternativos y se utilizaron 24 clases de los mismos colegios como controles. Las clases de control utilizaron los programas de texto básico de lectura adoptados en sus distritos escolares. El estudio duró un año escolar completo. Los niños de las clases experimentales pasaron la misma cantidad de tiempo en la enseñanza de lectura y lenguaje - noventa minutos diarios.

Se utilizó un diseño de investigación de grupo control, pretest y postest. El vocabulario y la comprensión lectora se evaluaron utilizando el test de lectura de Gates-MacGinitie, Nivel B, forma 1. Las habilidades del silabeo se evaluaron utilizando un test de silabeo con ochenta y una preguntas de elección múltiple elaborado por uno de los investigadores.

El test de silabeo se validó dos veces. Un test de silabeo compuesto de ochenta palabras sin sentido se pasó individualmente a los estudiantes de una clase de segundo curso. Los mismos estudiantes también realizaron un test de silabeo que medía las ciento treinta y cuatro competencias de silabeo. Las puntuaciones estaban altamente correlacionadas $(r=.81, p<$. 005.). Las correlaciones del postest de las puntuaciones en silabeo de casi 1.110 estudiantes con sus puntuaciones en el test de rendimiento de GatesMacGinitie produjeron una correlación de $.77(p<.001)$. Vimos pues que el test de silabeo era bastante fiable al utilizar una correlación test-retest como índice de fiabilidad $(r=.73)$.

Se obtuvieron puntuaciones en autoimagen utilizando «una escala de autoconcepto gráfica para niños, de preescolar a cuarto curso» elaborada por D. W. Felker, A. S. Bolea, y M. D. Barners. Las actitudes hacia la lectura se evaluaron con un test desarrollado y validado por el equipo investigador. Se validó el test mediante la correlación de las puntuaciones de los estudiantes en "actitudes hacia la lectura" con las evaluaciones que hacían de los profesores de las actitudes de los estudiantes. Se administraron todos 


\section{1}

los test durante la primera semana de septiembre de 1983 y la segunda semana de mayo de 1984.

\section{CINCO ESTRATEGIAS ALTERNATIVAS DE ENSEÑANZA}

Los investigadores desarrollaron cinco aproximaciones experimentales al desarrollo de la lectura. Se entrenó a los profesores en el uso de estas estrategias cuatro meses antes de su implementación en la clase.

\section{Alternativa 1: Programas sobre texto básico de lectura con agrupamiento homogéneo tradicional y 10-15 minutos del programa especial de decodificación}

La primera aproximación a la enseñanza de la lectura era idéntica a la de los programas con texto básico seguidos en las clases de control, con la excepción de que los profesores sustituían la instrucción en decodificación proporcionada en sus programas básicos por un programa especial de decodificación desarrollado por Eldredge (1984). Se instruyó a los profesores de forma que no utilizasen más de quince minutos diarios en esta instrucción de decodificación.

Se enseñó a los alumnos a reconocer los sonidos de veinte grupos vocálicos y aplicar tres principios básicos de silabeo para determinar el sonido de la vocal en palabras desconocidas antes de intentar vocalizarlas. Después de identificar el sonido vocálico de una palabra desconocida se le enseñaba a enlazar las letras consonantes precedentes a la vocal con el sonido vocálico, y finalmente a sintetizar todo con el sonido resultante. Por ejemplo, la mezcla o combinación sintética de la palabra inglesa «bat» sería $/ \mathrm{a} /$, /ba/, /t/, /bat/.

Se enseñó a los alumnos a utilizar tres normas de silabeo para su uso en palabras polisilabas aplicando las habilidades de silabeo que habían aprendido a sílabas individuales de palabras más largas. Se les enseñó a aplicar las estrategias de análisis estructural y de silabeo sólo cuando la utilización del contexto era ineficaz.

\section{Alternativa 2: Programas básicos con agrupamiento heterogéneo}

Se proporcionaba a todos los alumnos de este programa el mismo texto básico de segundo curso. Los profesores enseñaban las lecciones de destrezas señaladas en el manual del profesor e introducían el nuevo vocabulario para historias reuniendo al grupo de clase completo. Se asignaba a los alumnos trabajo de mesa de refuerzo, proporcionado por el programa del texto básico, tan pronto como se impartian las lecciones. Estas actividades debían ser terminadas después de que los estudiantes hubiesen leído la historia del libro de lectura básico.

Una vez presentada la historia, los estudiantes la leían desde el comienzo hasta el final sin que el profesor les interrumpiera. Los estudiantes que pudieran leer la historia en silencio sin ninguna ayuda eran asignados a un equipo de aprendizaje de tres alumnos. Estas tríadas eran heterogéneas. y cambiaban cada semana. Había al menos un buen lector en cada tríada.

Se seleccionaba un jefe de grupo para cada tríada de forma que contro- 
lase el progreso de todos los miembros del equipo. Si los alumnos necesitaban ayuda en una palabra determinada, tenían permitido buscar esa ayu$\mathrm{da}$ en cualquier miembro del equipo.

A los estudiantes incapaces de leer el material básico de forma independiente se les asignaba para que leyesen el material junto con otro alumno que utilizaba el Neurological Impress Method (Método de Impresión Neurológica). Los alumnos de estas díadas de aprendizaje también cambiaban cada semana.

El «Método de Impresión Neurológica», una forma de lectura oral al unísono, ha sido descrito por Heckelman (1966). El uso modificado utilizado en este estudio implicaba emparejar un mal lector con un lector mediano capaz de leer el material. Los estudiantes se sentaban uno junto a otro leyendo en voz alta de un libro. El estudiante más rápido señalaba cada palabra mientras la leía, y el estudiante más lento repetía cada palabra tras él. Se enseñaba al lector más rápido a leer el material con fluidez, evitando la lectura palabra a palabra. Se enseñaba al estudiante más lento a mirar las palabras conforme las iba leyendo el estudiante más rápido y a decirlas tan rápidamente como le fuese posible.

Según progresaba el mal lector, el estudiante que le ayudaba simplemente leía en silencio con él aportándole palabras sólo cuando le era necesario.

Para la discusión de la historia y la corrección del trabajo de mesa, los alumno agrupados en díadas se asignaban a una tríada. Los alumnos en díadas hacían juntos el trabajo de mesa. Los alumnos en tríadas hacían sus tareas independientemente tan pronto como terminaban de leer la historia.

Cuando la-el jefe de equipo consideraba que ya era hora de corregir la tarea, organizaba la discusión para que los miembros del equipo comentasen sus distintas respuestas. Se obtenía un acuerdo en las respuestas, lo que requería que algunos de los alumnos cambiasen sus contestaciones. Tras esta discusión, el jefe de equipo obtenía una copia de la hoja de respuestas del profesor y todas las tareas se corregían siguiendo esta pauta. Si un miembro del equipo fallaba una pregunta, todos la fallaban. Tras registrar las puntuaciones en la hoja de tareas, cada estudiante clasificaba su hoja personal de tareas en su archivador que se guardaba cerca del pupitre del profesor.

El jefe de equipo de cada tríada también dirigía una discusión de la historia con los miembros de su equipo después de que todos hubiesen acabado de leer la historia. El profesor había preparado con antelación las preguntas para esta discusión, que estaban en hojas de trabajo y sobre su mesa para que los jefes de equipo las cogiesen cuando estuviesen listos.

Tras la discusión de la historia cada miembro del equipo firmaba en la pizarra para hacer saber al profesor que había tenido lugar una discusión. El profesor organizaba entonces grupos de unos diez estudiantes para actividades adicionales de comprensión o para revisar objetivos, según se deseaba.

\section{Alternativa 3: Programas básicos con agrupamiento heterogéneo y 10-15 minutos de programa especial de decodificación}

Este programa era idéntico a la alternativa 2, con la excépción de que los estudiantes recibían el programa de decodificación especial ofrecido en la alternativa 1. 
Alternativa 4: Programa de literatura

Aquí los procedimientos eran similares a los aconsejados por Veatch (1984). La mayor parte del período de lectura se dividía en lectura de los niños, actividades para estimular su interés en la lectura, y lectura de libros en voz alta por parte de los profesores. Las actividadese de comprensión para desarrollar el vocabulario y habilidades de pensamiento se elaboraban mediante materiales que se leían a los niños, «sesiones de compartir el libro», y lecturas sobre temas del área de contenido.

Los niños seleccionaban los libros que querían leer. Aquellos que podían leer en silencio sin ayuda eran asignados a equipos de tres estudiantes (tríadas) similares a los descritos en las alternativas 2 y 3 . Aquellos que no podían leer los libros de forma independiente se emparejaban con estudiantes que si podían hacerlo. Estas parejas utilizaban el Método de Impresión Neurológica.

\section{Alternativa 5: Programa de literatura y programa de decodificación especial de 10-15 minutos.}

Este programa era idéntico al de la alternativa cuatro, excepto en que los estudiantes de este grupo recibían el programa de decodificación especial ofrecido en las alternativas uno y tres.

\section{LAS MEJORES ALTERNATIVAS: LA LITERATURA Y LA DECODIFICACION ESPECIAL}

La comparación de los cinco programas experimentales con sus respectivos controles indicó que estos tres programas, entre los experimentales, producían mejoras significativas con respecto a los programas tradicionales: el programa de literatura que utilizaba las estrategias de decodificación especial, el programa de literatura que no utilizaba dichas estrategias, y el grupo de programas tradicionales con texto básico que utilizaba las estrategias de decodificación especiales.

La comparación entre los cinco programas fundamentales puso de manifiesto veinte diferencias significativas. Catorce de ellas a favor del programa de literatura complementado con la instrucción de decodificación especial; cuatro aparecían a favor del programa de agrupamiento heterogéneo complementado con decodificación especial; y dos estaban a favor del programa de agrupamiento homogéneo apoyado con decodificación especial.

Los análisis estadísticos de los efectos de los materiales, del agrupamiento y de la decodificación, sobre el rendimiento y las actitudes hacia la lectura indicaron que: 1) El uso de literatura infantil para enseñar a leer a los niños tenía un efecto positivo sobre el rendimiento y actitudes hacia la lectura de los estudiantes - mucho mayor que los métodos tradicionales utilizados. 2) El uso de la instrucción en decodificación especial también tuvo un efecto positivo sobre el rendimiento y actitudes hacia la lectura de los alumnos mucho mayor que la instrucción de decodificación tradicional. 3) el uso del agrupamiento heterogéneo afectó positivamente el rendimiento (Eldredge y Butterfield, 1984). 
Los descubrimientos de este estudio ofrecen apoyo a los profesores que quieren utilizar los libros de literatura infantil para enseñar a leer a los niños. Se obtuvieron interesantes datos con 104 libros de literatura empleados en el estudio, promediando las puntuaciones a partir de doce fórmulas principales de amenidad; el $91 \%$ de los libros tenía puntuaciones superiores al nivel 4.0. Los estudiantes de segundo curso leían esos libros y disfrutaban con ellos a pesar de la falta de "controles de vocabulario" y la despreocupación por cribar las obras según la longitud de las frases. No sólo obtuvieron mejoras más importantes en rendimiento que los niños que utilizaban programas básicos de lectura, sino que sus actitudes hacia la lectura también mejoraron de forma significativa. Las actitudes hacia la lectura disminuyeron entre aquellos niños que utilizaban textos básicos de lectura durante este mismo período de tiempo. Los materiales sí producen diferencias.

Puede ser que los niños que disfrutan más leyendo, lean realmente más. Si es así, podría darse el caso de utilizar libros de literatura infantil bajo ese único criterio dado que hay estudios que sugieren que la cantidad de tiempo que los niños pasan leyendo en el colegio se asocia con ganancias en rendimiento y lectura (Allington, 1984; Leinhardt, Zigmond y Cooley, 1981).

Como promedio, los niños a los que se les enseña precozmente silabeo sistemático están dispuestos para un mejor comienzo en el aprendizaje de la lectura que los niños a los que no se les enseña silabeo (Chall, 1983; Johnson y Baumann, 1984; Pflaum et al., 1980, Williams, 1985). Este estudio apoya a aquellos que quieren explorar enfoques alternativos a la enseñanza de silabeo de los programas básicos de lectura.

Nuestro enfoque acentuó "la combinación consonántica». Los profesores que invierten cantidades de tiempo por encima de la media en combinación consonántica producen ganancias por encima del promedio en los test de rendimiento y lectura de primero y segundo curso. También sugiere que el silabeo explícito produce mejor rendimiento que el enfoque implícito de la mayoría de los programas básicos de lectura. En la instrucción del silabeo explícito, los sonidos asociados con las letras se identifican por separado y se combinan para formar palabras. En la instrucción de silabeo implítico el sonido asociado con una letra nunca se dice de forma aislada.

El mayor interés de la Comisión en cuanto al silabeo explícito radica en que tanto profesores como alumnos tienen dificultad en emitir sonidos aislados. Señala que el sonido "B" se convierte en /buh/ y que la combinación de los sonidos en la palabra "bat" puede resutar en /buh-ah-tuh/ dando como resultado que el niño no reconozca la palabra bat. El enfoque de decodificación utilizado en este estudio evitó este problema de combinación distorsionada dado que la vocal y la consonante que la precede se combinan juntas, dando como resultado una aproximación no deformada a la sintetización; p.e., $/ \mathrm{ba} / / \mathrm{t} /$.

\section{VALOR DEL AGRUPAMIENTO MIXTO}

Muchos estudios y revisiones de estudios han puesto de manifiesto los efectos negativos de agrupar a los alumnos por el criterio de capacidad de cara a la enseñanza de la lectura (Anderson et al., 1985; Hievert, 1983; Ku- 
lik y Kulik, 1982; Zakariya, 1985). Este estudio ofrece datos que apoyan la tesis de que los niños pueden aprender a leer en grupos heterogéneos tan bien como en grupos de capacidad homogénea. Nuestros resultados sobre el análisis de las respuestas de los profesores que utilizan estrategias de agrupamiento heterogéneo puede resumirse en lo dicho por uno de ellos: «nunca he disfrutaado más de la enseñanza de la lectura, ni he visto mejores resultados, ni he trabajado menos".

Los niños no tienen que sufrir la humillación de estar en un "grupo de lentos». Se les puede enseñar a leer en grupo heterogéneos igual de bien que en los típicos grupos de alta, media y baja capacidad. Una ventaja adicional a este agrupamiento alternativo es que puede requerir menos trabajo de los profesores.

Si los profesores y administradores quieren probar alternativas a la enseñanza tradicional de la lectura sin temor a pérdidas de rendimiento, cuentan con el apoyo de este estudio.

\section{Referencias}

Allington, RICHARD. «Oral Reading." In Handbook of Reading Research por P. David Pearson (ed.), Nueva York, N.Y.: Longman, 1984.

ANDERSON, RichARD C.; ElFRIEDA H. HiEbeRT; JUdITH A. SCOTT, y IAN. G. WilkinSON. Becoming a Nation of Readers: The Report of the Commission on Reading, Washington, D.C.: National Institute of Education, 1985.

Chall, JeANNE. Learning to Read: The Great Debate Nueva York, N.Y.: McGraw-Hill, 1983.

EldREDGE, J. Lloyd. Reading: A Structured Decoding Program. Provo, Utah: Brigham Young University Bookstone, 1984.

EldREDGE, J. Lloyd, y DeNNiE ButTERFIELD. Sacred Cows Make Good Hamburger: A Report on a Reading Research Project Titled "Testing the Sacred Cows in Reading." ED 255 861. Arlington, Va.: ERIC Document Reproduction Service, 1984.

Heckleman, R. G. "Using the Neurological Impress Remedial Reading Technique. Academic Therapy Quarterly, vol. 1 (verano 1966), pp. 235-39.

HIEBERT, ELFRIEDA. *An Examination of Ability Grouping for Reading Instruction.» Reading Research Quarterly, vol. 18 (invierno 1983), pp. 231-55.

JOHNSON, DALE D. y JAMES F. BAUMANN. "Word Identification.» In Handbook of Reading Research editado por David Pearson. Nueva York. N.Y.: Longman, 1984.

Kulik, Chen C. y James A. Kulik, «Effects of Ability Grouping on Secondary School Students: A Meta Analysis of Evaluation Findings. American Educational Research Journal, vol. 19 (otoño 1982), pp. 415-82.

LeinHARDT, GAEA; NAOMI ZigmoND y WILliam W. COOLEY. «Reading Instruction and lts Efects." American Educational Research Journal, vol. 18 (1981), pp. 343-61.

Perfetti, Charles A., y Allen M. Lesgold. *Coding and Comprehension in Skilled Reading and Implications for Readings Instruction: Theory and Practice of Early Reading editado por Lauren B. Resnick y Phyllis A. Weaver. Hillsdale, N. J.: Erlbaum, 1979.

Pfaum, Susana W.; Herbert Walberg; MYra L. Karegianes, y Sue P. Rasher. «Reading Instruction: A Quantitative Analysis.» Educational Researcher, vol. 9 (julio-agosto 1980). pp. 12-18.

ROSENSHINE, BARAK, y ROBERT STEVENS. *Classroom Instruction in Reading." In Handbook of Reading Research, editado por P. David Pearson. Nueva York. N.Y.: Longman, 1984.

VEALCH, JEANNETTE. Reading in the Elementary School Nueva York. N.Y.: R.C. Owen, 1984.

WILliams, JOANA P. *The Case for Explicit Decoding Instruction." In Reading Education: Foundations for a Literate America editado por Jean Osborn; Paul T. Wilson, y Richard C. Anderson. Lexington, Mass.: Lexington Books, 1985. 
Zakariya, Sally Banks. «To Boost Kids Reading Skills, Pack Away the Workbooks and Bring on the Books.» The American School Board Joumal, (agosto 1985). pp. 17-20.

Datos sobre los autores

En la Universidad Joven de Brigham Provo, Utah, Elredge imparte cursos de lectura para licenciados y estudiantes en el Departamento de Educación Básica, $y$ Butterfield da clases en el Departamento de Curriculum y Ciencias de la Educación.

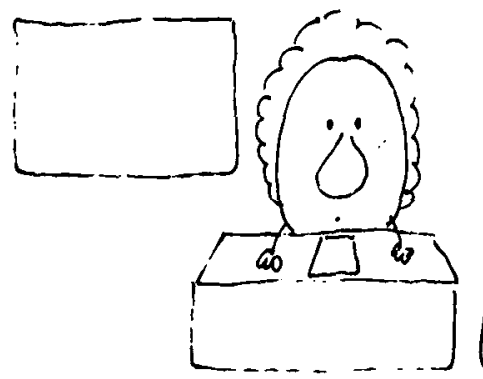

Seño, no lcomprendo las preguntas de la hoja de Comprension

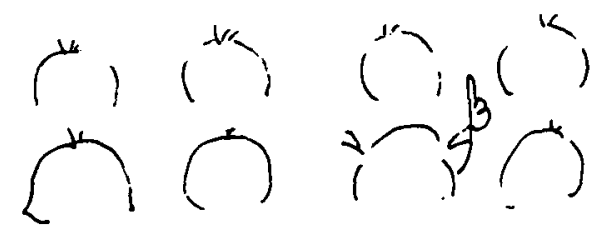

